\title{
The Scope of Adaptive Digital Games for Education
}

\author{
Rikki Prince, Hugh C. Davis \\ Learning Societies Lab \\ School of Electronics and Computer Science \\ University of Southampton \\ $\{r f p 07 r, h c d\} @ e c s . s o t o n . a c . u k$
}

\begin{abstract}
In learning technologies, there is a distinct difference between the user sequencing in a system based on IMS Simple Sequencing and an Adaptive Hypermedia system. This range of possibilities is important to consider when attempting to augment educational games with adaptive elements. This poster demonstrates how truly adaptive games could be designed and discusses why this is useful in the field of education.
\end{abstract}

\section{Introduction}

This poster presents the concept of fully adaptive digital games for education. Although there have been a number of research papers about educational digital games which adapt to the player, they could be considered quite reserved in the amount of adaptivity they incorporate.

Moreno-Ger et al. [10] demonstrated a system which, based on user information stored in a virtual learning environment (VLE), would select the most appropriate of three levels in the game for the user to start on.

A methodology for generating an environment made up of multiple games adaptively selected from a repository of games was presented by Carro et al. [4]

Conati and Zhao [5] reported on the results of experiments using their pedagogical agent. Their agent augments Prime Climb, which is a two player game used to improve understanding of number factorisation. The agent tracks the player's performance as a Dynamic Bayesian Network, and uses the statistics generated from it to select which of a set of hints to display to the user.

Some reasons why games are not yet adaptive, and how other research fields could inform the design of adaptive digital games is discussed in sections two and three.

Abdullah and Davis [1] presented the differences between using an IMS Simple Sequencing system and an Adaptive Hypermedia system. Importantly, they assert that Simple Sequencing is instructor centred while Adaptive Hypermedia is user centred.

The key differences between the two systems is in how they internally represent the material to be presented to the learner, how they model what the learner already knows and how they use those to adapt the material.

IMS Simple Sequencing [7] is a specification that uses a custom XML markup to organise learning activities in a tree hierarchy, so that the learner can either be guided on a particular route through the sub-nodes and leaves of the tree, or can be given complete freedom to navigate the tree. More intricate behaviours can be created by having conditional rules on the various nodes of the tree, which can enable the learner to skip certain leaves of the tree if they previously met certain objectives.

Adaptive Hypermedia has been an active research area since the early 1990s. Numerous architectures have been suggested for adaptive hypermedia systems, notably de Bra's Adaptive Hypermedia Architecture Model (AHAM) [2] and de Vrieze's Generic Adaptivity Model (GAM) [6]. There are three key components of these architectures. The domain model represents the material which can be presented to the user, in a way that the system understands so it can determine how to adapt it. The user model tracks what the user has done in the past and from this infers their preferences and future needs. The adaption model provides the rules that relate the information about the user to the domain model, and allows the adaption engine to create an experience unique to that user.

Brusilovsky's taxonomy [3] of adaptive hypermedia technologies sets the scope for what can be changed in an adaptive hypermedia system. Fragments of text can be inserted, removed or reordered. Links on a page can be hidden, sorted and annotated. There is even scope for new links and natural language fragments to be generated.

Section four is a discussion of the possible extents of adaption in digital games.

The motivation behind proposing adaptive digital games is to enhance learning and improve accessibility to 
educational games. Many adaptive hypermedia applications are in the educational domain, and it is hoped that adaptive games could have a similar impact.

Laurillard's Conversational Model [9] for tertiary education promotes a learning experience where the tutor can alter their explanation of a concept, the examples they use to demonstrate it and the problems they set the student to help them use the concept. However, the model is impractical when teaching a large number of students. The concept of the teacher changing what they present to the student, based on the student's apparent understanding of the topic at hand, bears a striking resemblance to the goals of an adaptive application.

Some of the reasons for using adaptive hypermedia could equally apply to adaptive games. For example, Kaplan suggests that HYPERFLEX, an adaptive hypertext system, could improve information retrieval time by up to $40 \%$ [8]. Presenting the correct educational material at the right time could prove invaluable in an educational game; otherwise the learner may unnecessarily spend hours playing the game to access what they need to learn.

\section{What is not an adaptive game?}

The typical modern digital game can be deconstructed into the software engine and the digital content.

The software engine is programming code compiled for the target platform and functions like most regular computer systems. It accepts some input: usually button or key presses, and mouse or joystick movements. These inputs are then processed by the engine by applying some logical rules that are typically specific to the game being played, which updates the internal game state. Finally, the engine produces some output, often including a rendered 3D scene, surround sound and sometimes haptic feedback.

The digital content includes just about everything that is not computer code. The visual output for a game is commonly a 3D scene rendered many times per second. This requires models, which represent the $3 \mathrm{D}$ shape, and textures, which form the material of the outer skin, for all the objects within the world. The audio landscape of a game is made up of pre-recorded music and sound effects. From these raw elements, the world is constructed and scripts written to tell a story within the game.

There are elements of adaptivity within some games already. Some games attempt to adjust the difficulty of the game based on the player's current performance. A simple example of this dynamic difficulty adjustment might be to change the number of opponents the player is faced with in the next stage, in relation to how quickly they dispatched with the opponents in the current stage.

Sandbox games, such as Grand Theft Auto, adapt to what the player has already done. In these games, the player has a choice of which tasks they attempt next, each leading along a slightly different part of the storyline. Rather than having every task available to the player from the start, the majority are only made available to the player based on the completion of other tasks.

However, once the rules and goals within a software engine are written and the art assets are created, the game is essentially fixed in this state. There are a finite number of routes through the game, so given enough time, a player can play the whole game enough to experience every possible storyline and view all of the content. In fact, any adaption that affects events over the whole length of the game are generally based on some very limited choice (of around 2-5 options), while most others are short lived and only affect the current situation the player is in.

\section{Towards Adaptive Games}

This all leads towards the question: how could a fully adaptive game be created? Having discussed the components of a game and their current adaptability, it is now possible to suggest what would be required to construct a truly adaptive game.

To begin with, the game engine would have to be built with total adaptivity in mind. Where the rules and goals of the game can typically be hardcoded into the program logic, the engine would need to support the ability to alter the rules or generate new ones on the fly. In addition, the engine could flexibly support different control systems (input) and feedback mechanisms (output). This would not only add a level of personalisation whereby the game could automatically identify the player's preferred method of interaction, but could also allow the game to adapt itself to use less common input devices, improving accessibility to players who cannot use a mouse, keyboard or control pad, while retaining the same basic game.

Content assets are traditionally hand crafted by artists, musicians, level designers and scriptwriters. This creates a fairly rigid game, with any illusion of adaptivity relying on a huge amount of pre-created assets, or clever blending of existing assets to create new ones (as is sometimes performed to create a new animation from two existing complimentary animations). A step towards a solution is the emerging technology of procedurally generated content. This approach involves designing an algorithm to generate a particular asset, in such a way that, by changing the parameter values which initialise the algorithm, a wide range of variations of the asset can be created.

Given the technologies to adapt both the functionality of the game engine and the content assets available, the concept of a fully adaptive game seems more feasible. The system would also require a way to decide what to adapt, when to adapt it and how to adapt it, and some data 
to base those decisions on. On this matter, inspiration can be sought in the field of adaptive hypermedia.

An adaptive game would require some form of model of the player. While some games can store the player's current state for future retrieval, and previous high scores, few store a persistent record of the past actions of the player. Microsoft's Xbox Live stores a form of user model, tracking all of the games a player has played and the 'achievements' they have completed, though this data is limited to games on the Xbox platform.

The next important element is a model of the content, or the parameters within which the content can be procedurally generated. Along with this, a set of rules which specify when and how to perform the adaption, is required. These elements are analogous to the domain and adaption models, respectively, in AHA and GAM.

Finally, it would be necessary to have some form of software engine to detect when the adaption rules are triggered, use the model of the player to decide what to adapt and then process the content into its new form. This would need to be fairly closely integrated with, or else have a well defined interface to, the game engine.

\section{Discussion}

What, then, is the range of possible adaption in digital games? Can a line be drawn to divide a system which is truly adaptive and one which is just a rearrangement of existing content, based on a finite rule set?

This question somewhat alludes to the answer. If an application just chooses a different order or skips certain segments, based on exposure and performance in previous parts, then it seems it is not fulfilling its adaptivity potential. The information within the segments could be adapted or even generated from scratch to suit the user.

Another area in which a truly adaptive system excels is its user modelling. By storing more details about the player's interactions, previous experience and preferences, there is more data with which the engine can tune the adaption, and potentially improve the player's experience.

\section{Conclusion}

The concept of fully adaptive digital games for education has been presented here. While existing games have a fairly rigid structure in terms of the rules governing the game and the art content available, a possible design for making games highly adaptive has been suggested.

The scope of adaptability within various forms of adaptive games was discussed, resulting in the conclusion that detailed user modelling and the ability to alter and generate new content based on the model of the user, are key to a fully adaptive system.
Future work on this research will see an attempt to construct a functioning adaptive educational digital game engine, to demonstrate that these ideas are valid.

The concept of an adaptive digital game raises many more questions. How could an adaptive system ensure the game remains fair and balanced? How would an adaptive game affect replayability? How can a tutor author material for an adaptive game?

\section{References}

[1] N. A. Abdullah and H. Davis, "Is simple sequencing simple adaptive hypermedia?" in HYPERTEXT '03: Proceedings of the fourteenth ACM conference on Hypertext and hypermedia, ACM, New York, 2003, pp. 172-173.

[2] P. De Bra, A. Aerts, B. Berden, B. de Lange, B. Rousseau, T. Santic, D. Smits, and N. Stash, "AHA! the adaptive hypermedia architecture," in HYPERTEXT '03: Proceedings of the fourteenth ACM conference on Hypertext and hypermedia, ACM, New York, 2003, pp. 81-84.

[3] P. Brusilovsky, "Adaptive hypermedia," User Modeling and User-Adapted Interaction, vol. 11, no. 1-2, pp. 87-110, 2001.

[4] R. M. Carro, A. M. Breda, G. Castillo, and A. L. Bajuelos, "A methodology for developing adaptive educational-game environments," in Adaptive Hypermedia and Adaptive WebBased Systems, Springer, Berlin, 2002, pp. 90-99.

[5] C. Conati and X. Zhao, "Building and evaluating an intelligent pedagogical agent to improve the effectiveness of an educational game," in IUI '04: Proceedings of the 9th international conference on Intelligent user interfaces. ACM, New York, 2004, pp. 6-13.

[6] P. de Vrieze, P. van Bommel, and T. van der Weide, "A generic adaptivity model in adaptive hypermedia," in Adaptive Hypermedia and Adaptive Web-Based Systems, Springer, Berlin, 2004, pp. 344-347.

[7] IMS GLOBAL LEARNING CONSORTIUM. IMS Simple Sequencing best practice and implementation guide. Electronic, March 2003. [Online]. Available: http://www.imsglobal.org/ simplesequencing/ssv1p0/imsss bestv1p0.html.

[8] C. Kaplan, J. Fenwick, and J. Chen, "Adaptive hypertext navigation based on user goals and context," User Modeling and User-Adapted Interaction, vol. 3, no. 3, 1993, pp. 193-220.

[9] D. Laurillard, Rethinking University Teaching: A Framework for the Effective Use of Educational Technology, Routledge, 1993.

[10] P. Moreno-Ger, D. Burgos, J. Sierra, and B. Manjón, "A game-based adaptive unit of learning with IMS Learning Sesign and <e-Adventure>," in Creating New Learning Experiences on a Global Scale, Springer, Berlin, 2007, pp. 247-261. 